\title{
REVIEW
}

\section{Inhibitors of histone-deacetylase}

\author{
Jiřina Vávrová ${ }^{1}$, Martina Řezáčová ${ }^{2}$, Jan Osterreicher ${ }^{1}$ \\ ${ }^{1}$ Department of Radiobiology, School of Military Health Sciences Hradec Králové, University of Defence Brno, \\ Třebešská 1575, 50001 Hradec Králové, Czech Republic \\ ${ }^{2}$ Institute of Medical Biochemistry, Faculty of Medicine Hradec Králové, Charles University Praha, \\ Šimkova 870, 50001 Hradec Králové, Czech Republic
}

Received $24^{\text {th }}$ January 2005.

Revised $14^{\text {th }}$ April 2005.

Published online $25^{\text {th }}$ May 2005.

\begin{abstract}
Summary
Histone acetyltransferase and histone deacetylase activities determine the acetylation status of histones, and have the ability to regulate gene expression through chromatin remodeling. A controlled balance between histone acetylation and histone deacetylation appears to be essential for normal cell growth. In cancer cells, some genes are repressed by inappropriate recruitment of histone deacetylases. The histone deacetylase inhibitors (HDACI) belong to the class of anticancer drugs that are effective in killing proliferating and non-proliferating tumor cells. In this review we discuss molecular mechanisms involved in the induction of cell cycle arrest, differentiation and induction of apoptosis in tumor cells by HDACI.
\end{abstract}

Keywords: histone deacetylase inhibitors - apoptosis - cell cycle - chemotherapy

\section{INTRODUCTION: THE ROLE OF HISTONES IN DNA STRUCTURE AND FUNCTION}

Recent extensive studies have revealed the essential role of the chromosome structure in the regulation of transcription. In eukaryotic cells, transcription is affected by the mode of DNA coiling. At least one functional consequence of chromatin packaging is to prevent access of DNA-binding proteins thereby regulating transcription to the promoter. The basic unit of chromatin is nucleosome.

Jiřina Vávrová, Katedra radiobiologie, Fakulta vojenského zdravotnictví, Třebešská 1575, 50001 Hradec Králové, Czech Republic

ㅁarova@pmfhk.cz
Nucleosome is organized around a histone octamer containing two molecules each of core histones $\mathrm{H} 2 \mathrm{~A}, \mathrm{H} 2 \mathrm{~B}, \mathrm{H} 3$ and $\mathrm{H} 4$. The histone octamer forms the protein core of the nucleosome, which is entwined by 146 base pairs of DNA. Histone $\mathrm{H} 1$ associates with chromatin outside the nucleosome and regulates higher order chromatin structure. So in the nucleosome, two turns - 166 base pairs - of the DNA are super coiled in a lefthanded helix over the surface of the histone octamer (central H3-H4 tetramer and two H2A$\mathrm{H} 2 \mathrm{~B}$ dimmers), sealed by histone H1. The local architecture of chromatin is an important factor of gene expression regulations. During activation of the gene transcription the tight chromatin structure is released by nucleosome modifications, thus enabling the binding of transcription proteins. Two crucial mechanisms are involved in chromosome 
structure alterations: (ii) post-translational modification of histones and (ii) ATP-dependant remodeling of chromosomes (Luo and Dean 1999).

The terminal parts of histones are subject to five types of post-translational covalent modifications: acetylation, methylation, phosphorylation, ADP-ribosylation, and covalent linkage (H2A) to ubiquitin (Luo and Dean 1999, Davie 1998). The most frequently studied histone modification is acetylation, which is linked mainly to the transcriptional activation of chromatin, while histone deacetylation is linked to transcriptional repression. Acetylation occurs mainly on lysine residues of N-terminus of core histones, such as lysine 8,12 or 16 of $\mathrm{H} 4$, or lysine 9, 14 or 23 of $\mathrm{H} 3$. Acetylation of histones is regulated by two groups of enzymes - histone-acetyl-transferases (HAT) and histone-deacetylases (HDAC). In steady state a dynamic equilibrium is reached between HAT and HDAC activities. The dominance of HDAC activity causes deacetylation leading to a highly organized chromatin structure and inhibition of transcription.

\section{HISTONE-DEACETYLASES}

Members of the classic HDAC family fall into two different phylogenetic classes, namely class I and class II. The class 1 HDAC (1, 2, 3 and 8) are most closely related to the yeast (Saccharomyces cerevisiae) transcriptional regulator RPD3. Class II $\operatorname{HDAC}(4,5,6,7,9$ and 10) share domains similar to HDA1, another deacetylase found in yeast (de Ruijter et al. 2003). While class I HDAC are generally expressed in most cells, the expression of class II HDAC is predominantly related to an activated process of cellular differentiation and development. To exert their function, HDAC need to be in the nucleus, where the predominant substrate is found. Class I HDAC are found mostly in the nucleus. While HDAC1, 2 and 8 are localized exclusively in the nucleus, HDAC3 are situated mainly in the nucleus, but after specific signals they can be probably relocated also to the cytoplasm, as it has signals for nuclear export and import. The class II HDAC can be transported between the nucleus and the cytoplasm in response to certain signals. It has been found that HDAC 4, 5 and 7 play a crucial role in the regulation of proliferation and differentiation of muscle cells. Prior to muscle differentiation, these HDAC are localized in the nucleus, during differentiation they are relocalized to the cytosol and, after differentiation, HDAC4 returns to the nucleus. This process is regulated by $\mathrm{Ca}^{2+} /$ calmodulin dependent kinase (phosphorylation of HDAC), CRM1 (cellular export factor), and 14-3-3 protein (cytosolic anchor protein). Using SAGE (serial analysis of gene expression) data from the Human Transcriptome Map (Caron et al.
2001), De Ruijter et al. (2003) found that HDAC 1, $2,3,5,6,7$ and 10 are more or less generally expressed in all tissues examined (brain, breast, colon, kidney, ovary, pancreas, prostate, heart). HDAC 4, 8 and 9 can be found predominantly in tumor cells.

It is not entirely clear how histone acetylation contributes to increased sensitivity of DNA to transcription factors. The first assumption was that the modification of the basic $\varepsilon$-aminogroup of lysines on $\mathrm{N}$-terminus of histones by the acidic acetyl group neutralizes the basic character of histones and decreases the histone affinity to DNA. However, it is more likely that the acetylation of $\mathrm{H} 3$ and $\mathrm{H} 4 \mathrm{~N}$-terminal parts interferes with the highly organized structure of chromatin, but does not destabilize histone-DNA interactions in nucleosome. The histone N-terminal tails, as defined from analysis of the crystal structure, are the regions of the histone protein sequences that reach outside of the nucleosomal disk and their direct interaction with DNA is minute (Luger and Richmond 1998). It is unlikely that the acetylation of a couple of basic lysine residues in the histone tails could exert great changes in nucleosome structure, however, these tails play an important role in the rearrangement of nucleosomes into a higher-order structure and in the access of some transcription factors to nucleosomal DNA.

\section{INHIBITORS OF HDAC}

It has been found that defects of status of histone acetylation are related to the expression of many cellular oncogens and tumor-suppressor genes. These findings have initiated an extensive search for substances capable of HDAC inhibition. One of the current draft hypotheses is that HDACI (histone deacetylase inhibitors) can reactivate the expression of tumor-suppressor genes, which is often suppressed in tumor cells. Rosato et al. (2004) classified HDACI into four groups according to their chemical structure:

1. short chain carboxylic acids, such as butyrate $(\mathrm{BuA})$, phenylbutyrate (PB), phenylacetate (PA), valproic acid (VA);

2. synthetic benzamid derivates, such as MS-275, CI-994, N-acetyldinaline;

3. cyclic tetrapeptides, such as apicidin, trapoxin; and

4. hydroxamic acids, such as suberoylanilid hydroxamic acid (SAHA), trichostatin A (TSA), azelaic bishydroxamic acid (ABHA).

Most of the hydroxamic acids as well as TSA cause histone hyperacetylation in very low concentrations (nanomolar to micromolar) (Yoshida et al. 1990). HDACI from carboxylic acid group are less potent 
(effective concentrations being millimolar), but some of them have been already clinically proven (VA and PB in epilepsy treatment). $1 \mathrm{mM} \mathrm{VA}$ causes acetylation of histones $\mathrm{H} 3$ and $\mathrm{H} 4$ in teratocarcinoma cells F9 over 12 hours (Gottlicher et al. 2001). HDACI can cause cell cycle arrest and commitment of cells to differentiation or apoptosis (Yoshida et al. 2001). The relation between acetylation and transcription can be proved by treatment of mammalian cells by HDACI. The result of HDACI function is histone hyperacetylation, which is, after the relaxation of the chromatin structure, followed by transcriptional activation of some genes. For example Suenaga et al. (2002) proved that TSA and BuA decrease telomerase activity in prostate cancer cells by suppression of the catalytic subunit of telomerase (hTERT).

\section{EFFECT OF HDACI ON CELLULAR PROCESSES}

Acetylation of histones represents a crucial mechanism for the control of chromatin remodeling and gene regulations. The major interest concentrates on genes regulating DNA synthesis, cell cycle and apoptosis. Glaser et al. (2003) studied gene expression in tumor cell lines T24 bladder and MDA breast carcinoma after treatment with three HDACI: SAHA, TSA and MS-275. Despite the widely different structure of all three HDACI used, the changes in gene expression were very similar, which shows that these changes are related to the mechanism of the action of the substances studied and not with their structure. Only 13 genes (less than $10 \%$ of studied genes) were changed, the expression of 5 genes decreased and 8 increased. One of the genes whose expression increased was the gene for $\mathrm{p} 21$. This observation is supported by the increase of $\mathrm{p} 21$ protein in reaction to HDACI, detected by western blot analysis in other studies (Blagosklonny et al. 2002, Gui et al. 2004, Burgess et al. 2001, Kim et al. 2003, Rosato et al. 2004). Also the other regulator of the cell cycle - p27 increased in response to HDACI.

Also Ragione et al. (2001) proved (using the DNA micro-array method) that HDACI induce changes of only a small number of genes $(1-7 \%)$ in the colon carcinoma cell line HT-29. They compared changes of gene expression after exposure to two HDACI - BuA and TSA. All experiments were performed in the presence of cycloheximide, to allow observations of direct transcriptional effects only. They found that both agents influence identical gene groups, indicating the importance of the inhibition of HDAC. Decreased expression was found only in two genes, the other 21 genes showed increased expression. A number of genes with increased expression (tob-1, GATA-2, GADD45, PKC $\delta$ ) control cell cycle regulation and signal transduction, DNA reparation and genome transcription. The expression of two of these genes - GATA-2 and tob-1 - increased more than 10fold. GATA-2 is the nuclear transcriptional factor, which is released in large quantities during tissue differentiation of cells. GATA protein level increase was also confirmed after incubation with BuA. The second gene analyzed - tob-1 - is an antiproliferative factor related to terminal differentiation of muscle cells, which was also found to increase during differentiation of osteoblasts (Beck et al. 2001). A Tob-1 protein level increase in the protein expression level was also confirmed after incubation with BuA. Also Glaser et al. (2003) described an increased expression of tob1 gene after incubation of T24 cells with SAHA and TSA, but not after MS-275. One of possible functions of tob-1 is an infliction of cell cycle arrest in $\mathrm{G}_{1}$ phase in the absence of $\mathrm{p} 21$. Table 1 summarizes the increase of gene expression in colon cancer cells HT-29 after incubation with BuA and TSA (Ragione et al. 2001) and in bladder carcinoma cells T24 after incubation with TSA, SAHA a MS-275 (Glaser et al. 2003).

Richon et al. (2000) found that the accumulation of acetylated histone at T24 bladder carcinoma cells is enhanced by cultivation with SAHA and that the increase of acetylation of histones localized to p21 gene is transient and correlates well with the transcriptional activity of the gene. Further, it was proved that HDACI cause induction of $\mathrm{p} 21$ and cell cycle arrest in $\mathrm{G}_{1}$ phase at Jurkat cells with mutated p53 (Blagosklonny et al. 2002) and in U937 cells without functional p53 (Vrana et al. 1999). Considering that many tumor cells lack functional protein $\mathrm{p} 53$ or $\mathrm{Rb}$ protein and therefore do not react to DNA damage by cell cycle arrest in $\mathrm{G}_{1}$ phase, induction of p21, p27 or tob-1 by HDACI provokes aberrant cell cycle arrest and can direct the cells to apoptosis (Glaser et al. 2003).

Cell cycle arrest or inhibition of proliferation can cause defects of DNA synthetic machinery, i.e. inhibition of thymidylate synthetase and CTP synthetase, probably by induction of apoptotic genes, e.g. n-clusterin. Clusterins are proteins induced in mammalian cells under stress. The role of clusterins in cell death processes is also complex and may be different for the various forms of the protein. While n-clusterin is an important factor for apoptosis induction at tumor cells, s-clusterin has a radioprotective effect. Nuclear clusterin is an ionizing radiation-inducible protein that binds $\mathrm{Ku}$ 70 (a protein important in reparation of double strand breaks of DNA after irradiation) and triggers apoptosis when over-expressed in MCF-7 cells (Leskov et al. 2003). Also incubation of T24 bladder carcinoma cells with HDACI SAHA caused 
a 15 fold increase in expression of this gene (Glaser et al. 2003).

The importance of cell cycle regulations after HDACI azelaic bishydroxamic acid was studied by Qiu et al. (2000). They found that there is no $G_{2}$ phase cell cycle arrest in ABHA sensitive cell lines (HeLa) after incubation with a relatively high concentration $(100 \mu \mathrm{g} / \mathrm{ml})$ of ABHA. The cells enter aberrant mitosis and are more sensitive to apoptosis induction in comparison to primary cultures of neonatal foreskin fibroblasts. Burgess et al. (2001) evaluated the anti-proliferative effect of relatively low concentrations $(10 \mu \mathrm{g} / \mathrm{ml})$ of ABHA. After this concentration apoptosis was not induced. They proved that the anti-proliferative effect of ABHA is related to the expression of p21. Cells, which do not react to ABHA by increase of $\mathrm{p} 21$, are more ABHA sensitive and undergo apoptosis after ABHA treatment. Experiments with isogenic cells $\mathrm{p} 21^{+/+}$and $\mathrm{p} 21^{-/-}$proved that expression of $\mathrm{p} 21$ reduces sensitivity to ABHA induced apoptosis.

Table 1. Human genes induced by HDACI

\begin{tabular}{|c|c|c|c|c|}
\hline Description & cells & HDACI & fold induction & function \\
\hline p21 & $\begin{array}{l}\text { HT-29 } \\
\text { T24 }\end{array}$ & $\begin{array}{l}\text { BuA, TSA SAHA, } \\
\text { TSA, MS- } 275\end{array}$ & $\begin{array}{l}4-5 \\
4-26\end{array}$ & cell cycle regulator \\
\hline GATA-2 & HT-29 & $\mathrm{BuA}, \mathrm{TSA}$ & $13-16$ & transcription factor \\
\hline TOB-1 & HT-29 & $\mathrm{BuA}, \mathrm{TSA}$ & $10-12$ & cell cycle regulator \\
\hline HM89 & HT-29 & BuA, TSA & 10 & chemokine receptor \\
\hline $\operatorname{PKC} \delta$ & HT-29 & $\mathrm{BuA}, \mathrm{TSA}$ & $9-10$ & $\begin{array}{l}\text { transduction } \\
\text { modulator }\end{array}$ \\
\hline EPH3 & HT-29 & $\mathrm{BuA}, \mathrm{TSA}$ & $7-8$ & $\begin{array}{l}\text { transduction } \\
\text { modulator }\end{array}$ \\
\hline GADD45 & HT-29 & $\mathrm{BuA}, \mathrm{TSA}$ & 3 & cell cycle regulator \\
\hline Нep27 & $\mathrm{T} 24$ & SAHA, TSA, MS-275 & $12-48$ & $\begin{array}{l}\text { short-chain alcohol } \\
\text { dehydrogenase }\end{array}$ \\
\hline TRPM-2 & $\mathrm{T} 24$ & SAHA, TSA, MS-275 & $12-23$ & clusterin-apoptosis \\
\hline alfa-tubulin & $\mathrm{T}-24$ & SAHA, TSA, MS-275 & 3 & cytoskeletal structure \\
\hline
\end{tabular}

During experiments analyzing gene changes in response to HDACI, it was found that some differences exist between different HDACI. It was proved that some MS-275-induced gene changes are different compared to changes induced by TSA and SAHA. MS-275 does not cause accumulation of acetylated tubulin alpha and also has no affect on the cell cycle regulator tob- 1 . Acetylation of tubulin is probably regulated by HDAC 6. Differences in the effect of a particular HDACI on this deacetylase can be important for changes in expression of some genes (Glaser et al. 2003). Blagosklonny et al. (2002) also detected differences of tubulin acetylation in response to TSA and $\mathrm{BuA}$, however, p21 increased after applications of both HDACI. In contrast to $\mathrm{BuA}$, TSA induced important tubulin acetylation during 16 hours and this increased acetylation correlates with apoptosis induction. It is interesting that tubulin acetylation after TSA treatment of Jurkat cells was increased as soon as 1 hour after beginning of incubation, but increase of p21 was observed only 8 hours after the beginning of incubation.

\section{INTERACTION OF HDACI WITH TUMOR CELLS}

Interactions of HDACI with tumor cells include many mechanisms, such as cell cycle arrest, influencing of differentiation and induction of cell death and thus, HDACI represent a new group of antitumor agents (Kim et al. 1999, Burgess et al. 2001). In low concentrations they have a cytostatic effect characterized by cell cycle arrest in $G_{1}$ phase and induction of inhibitor of cyclin dependent kinases protein $\mathrm{p} 21$. Cytostatic activity is not limited to tumor cells, as the normal cells are, after incubation with HDACI, also arrested in $\mathrm{G}_{1}$ phase (Qiu et al. 2000). At higher concentrations these compounds are selectively cytotoxic to tumor cells. 
Qiu et al. (2000) proved that the selective cytotoxicity is related to the absence of $\mathrm{G}_{2}$ arrest. The cells enter mitosis with non-repaired DNA and this aberrant mitosis is followed by cell death. Also the cells arrested in the $G_{1}$ phase die after 48-72 hours of incubation with HDACI, which suggest that HDACI can also kill non-proliferating cells. Burgess et al. (2004) proved that HDACI can kill proliferating and non-proliferating immortalized tumor cells, but that normal cells are not sensitive to these agents. The main difference between proliferating and non-proliferating cells is the time of death. In non-proliferating cells, there is a delay between exposure and death. It has been found that HDACI can kill tumor cells by two distinct pathways - the quick pathway, where the cells enter aberrant mitosis via defective $\mathrm{G}_{2}$ phase arrest, and the slow pathway through G1 phase arrest, p21 accumulation and slow apoptosis. An interesting fact is that p21 itself can inhibit the commitment of cells to apoptosis and therefore a decrease of p21 expression could induce apoptosis more quickly. Nebbioso et al. (2005) report that HDACI induce, in addition to $\mathrm{p} 21$, the expression of TRAIL, by direct activation of the TNFSF10 promoter, thereby triggering tumor-selective death signaling in acute myeloid leukemia (AML) cells and the blasts of individuals with AML. The results of Nebbioso et al (2005) identify TRAIL as a mediator of the anticancer activity of HDACI.

The overall destruction of tumor cells is the main goal of chemotherapy, however, many chemotherapeutical compounds destroy only proliferating tumor cells along with many proliferating stem cells of healthy tissues, which for the organism means a high overall toxicity of most chemotherapeutics. Burgess et al. (2004) proved that HDACI are selectively toxic to both proliferating and non-proliferating tumor cells. This ability makes HDACI very attractive for clinical studies and use.

\section{CLINICAL STUDIES}

Many studies (Clausen 1995, Jacobs 1988) proved that dietary factors play an important role in colon cancer development. It seems that the process responsible for cancer development protection is related to microbial fermentation of dietary fiber to short chain fatty acids, such as in BuA found naturally in the gastrointestinal tract. When administered at concentrations similar to those encountered within the colonic lumen, BuA causes growth inhibition, differentiation and apoptosis in a variety of colon cancer cell lines as well as in animal models (McIntyre et al. 1993, Wu et al. 2001, Rosato and Grant 2004). Hofmanová et al. (2005) proved increased apoptosis induction in human colon adenocarcinoma cells HT-29 after application of $\mathrm{BuA}$ and polyunsaturated fatty acid. BuA caused a similar effect when applied to human leukemia cells, where it potentiated differentiation and growth inhibition. Warell et al. (1998) successfully used sodium phenylbutyrate in therapy of promyelocytic leukemia resistant all-transretinoic acid (ATRA). The patient proved clinically resistant to treatment with ATRA alone. Over the next 23 days, when treated with both ATRA and $\mathrm{PB}$, visible leukemic cells were eliminated from the bone marrow. With a second treatment course, analysis for minimal residual disease by RT-PCR proved negative.

Valproic acid (VA) is a well-known compound used in the clinical long term therapy of epilepsy. Valproic acid and its derivates belong to a frequently used group of anti-epileptic drugs. They are used as a first choice treatment for partial attacks with simple symptomatology. It has been found that VA also posses HDACI activity and in vitro induces differentiation of tumor cells, transformed hematopoietic progenitors, and leukemic blasts from patients suffering from acute myeloid leukemia (Gotlicher 2004). In comparison to other HDACI VA induces inactivation of not only HDAC I, but also of HDAC II. VA is effective also in animal in vivo systems. However, VA is effective only in some specific tumor cells. Clinical trials are performed mainly on hematological malignancies and some solid tumors. New possibilities arise in the usage of the combined effect of VA and other substances, for example ATRA (Gottlicher et al. 2001). Kuendgen et al. (2004) treated 18 patients with the myelodysplastic syndrome (MDS) and AML secondary to MDS by VA monotherapy (concentration in serum was 346$693 \mu \mathrm{M})$. They conclude that VA is therapeutically beneficial for MDS patients, and ATRA may be effective when added later.

From the group of hydroxamic acid derivates the most prospective for clinical use seems to be SAHA. SAHA was effective as a differentiating and apoptosis-inducing agent in some tumor models, including prostate carcinoma, urinary bladder carcinoma, and myeloma (Butler et al. 2000). The activity of SAHA is related to the increase of protein p21 by the p53 independent pathway, which involves truncation of bid and generation of reactive oxygen species (ROS) (Vrana et al. 1999). The maximal tolerated dose of SAHA for hematologic patients was $300 \mathrm{mg} / \mathrm{m}^{2} /$ day for five days in three weeks. A daily intravenous application was well tolerated and histone acetylation was clearly apparent, with a half-life from 21 to 58 minutes. SAHA had anti-tumor activity in both solid and hematological tumors (Kelly et al. 2003).

HDACI are substances capable of induction of differentiation and apoptosis of tumor cells. They 
can partially modulate the expression of genes responsible for tumor cell proliferation. Combinations of HDACI and other substances seem to be important in anti-tumor therapy. For treatment of leukemias it is the combination of VA and ATRA (Kuendgen et al. 2004) or the combination of TRAIL (tumor necrosis apoptosis inducing ligand) and $1 \mathrm{mM} \mathrm{BuA}$ or $2 \mu \mathrm{M}$ SAHA, that cause activation of mitochondrial pathway of apoptosis induction and activation of caspases (Rosato et al. 2003). HDACI represent a novel strategy in chemotherapy.

\section{ACKNOWLEDGEMENTS}

The authors would like to thank the Grant Agency of the Czech Republic (grant project no. 202/04/0598) for financial support

\section{REFERENCES}

Beck G.R.Jr., Zerler B., Moran E.: Gene array analysis of osteoblast differentiation. Cell Growth Differ. 12:61-83, 2001.

Blagosklonny M.V., Robey R., Sackett D.L. et al.: Histone deacetylase inhibitors all induce p21 but differentially cause tubulin acetylation, mitotic arrest and cytotoxicity. Mol. Cancer Therap. 11:937-941, 2002.

Burgess A.J, Pavey S., Warrener R. et al.: Upregulation of $21^{\text {wafl/CIP1 }}$ by histone deacetylase inhibitors reduces their cytotoxicity. Mol. Pharmacol. 60:828-837, 2001.

Burgess A., Ruefli A., Beamish H. et al.: Histone deacetylase inhibitors specifically kill nonproliferating tumour cells. Oncogene 23:6693-6701, 2004.

Butler L.M., Agus D.B., Scher H.I. et al.: Suberoylanilide hydroxamic acid, an inhibitor of histone deacetylase, suppresses the growth of prostate cancer cells in vitro and in vivo. Cancer Res. 60:5165-5170, 2000.

Caron H., van Schaik B., van der Mee M. et al.: The human transcriptome map: Clustering of highly expressed genes in chromosomal domains. Science 291:1289-1292, 2001.

Clausen M.R.: Butyrate and colorectal cancer in animals and in humans (mini-symposium: Butyrate and colorectal cancer). Eur. J. Cancer Prev. 4:483-90, 1995.

Davie J.R.: Covalent modifications of histones: expression from chromatin templates. Curr. Opin. Genet. Dev. 8:173-178, 1998. de Ruijter A.J., van Gennip A.H., Caron H.N. et al.: Histone deacetylases (HDACs): characterization of the classical HDAC family. Biochem. J. 370:737-749, 2003.

Glaser K.B., Staver M.J., Waring J.F. et al.: Gene expression profiling of multiple histone deacetylase (HDAC) inhibitors. Defining a common gene set produced by HDAC inhibition in T24 and MDA carcinoma cell lines. Mol. Cancer Ther. 2:151-163, 2003.

Gottlicher M.: Valproic acid: an old drug newly discovered as inhibitor of histone deacetylases. Ann. Hematol. 83:S91-92, 2004.

Gottlicher M., Minucci S., Zhu P. et al.: Valproic acid defines a novel class of HDAC inhibitors inducing differentiatiation of transformed cells. EMBO J. 20:6969-6978, 2001.

Gui C.Y., Ngo L., Xu W.S., et al.: Histone deacetylase (HDAC) inhibitor activation of p21 WAF1 involves changes in promotorassociated proteins, including HDAC1. PNAS 101:1241-1246, 2004.

Jacobs R.: Role of dietary factors in cell replication and colon cancer. Am. J. Clin. Nutr. 48 (Suppl 3 ):775-779, 1988.

Hofmanová J., Vaculová A., Kozubík A.: Polyunsaturated fatty acids sensitize human colon adenocarcinoma HT-29 cells to death receptor-mediated apoptosis. Cancer Lett. 218:33-41, 2005.

Kelly W.K., Richon V.M., O'Connor O. et al.: Phase I clinical trial of histone deacetylase inhibitor: suberoylanilide hydroxamic acid administered intravenously. Clin. Cancer Res. 9:3578-3588, 2003.

Kim Y.K., Han J.W., Woo Y.N. et al.: Expression of p21 Waf1/Cip1 through $\mathrm{Sp} 1$ sites by histone deacetylase inhibitor apicidin requires PI 3kinase-PKC $\delta$ signaling pathway. Oncogene 22:6023-6031, 2003.

Kuendgen A., Strupp C., Aivado M. et al.: Treatment of myelodysplastic syndromes with valproic acid alone or in combination with alltrans retinoic acid. Blood 104:1266-1269, 2004.

Leskov K.S., Klokov D.Y., Li J. et al.: Synthesis and functional analyses of nuclear clusterin, a cell death protein. J. Biol. Chem. 278:1159011600, 2003.

Luo R.X., Dean D.C.: Chromatin remodeling and transcriptional regulation. J. Natl. Cancer Inst. 91:1288-1294, 1999.

Luger K., Richmond TJ.: The histone tails of the nucleosome. Curr. Opin. Genet. Dev. 8:140146, 1998.

McIntyre A, Gibson P.R., Young G.P.: Butyrate production from dietary fibre and protection 
against large bowel cancer in a rat model. Gut 34:386-391, 1993.

Nebbioso A., Clarke N., Voltz E. et al.: Tumorselective action of HDAC inhibitors involves TRAIL induction in acute myeloid leukemia cells. Nature Med. 11:77-84, 2005.

Qiu L, Burgess A., Fairlie D.P. et al.: Histone deacetylase inhibitors trigger a G2 checkpoint in normal cells that is defective in tumor cells. Mol. Biol. Cell 11:2069-2083, 2000.

Ragione F.D., Criniti V., Pietra V.D. et al.: Genes modulated by histone acetylation as new effectors of butyrate activity. FEBS Lett. 499:199-204, 2001.

Richon V.M., Sandhoff T.W., Rifkind R.A. et al.: Histone deacetylase inhibitor selectively induces p21 WAF1 expression and gene associated histone acetylation. PNAS 97:10014-10019, 2000.

Rosato R.R., Almenara J.A., Yu C. et al.: Simultaneous activation of the intrinsic and extrinsic pathways by HDAC inhibitors and TRAIL synergistically induces mitochondrial damage and apoptosis in human leukemia cells. Mol. Cancer Ther. 2:1273-1284, 2003.

Rosato R.R., Grant S.: Histone deacetylase inhibitors in clinical development. Expert Opin. Investig. Drugs 13:21-38, 2004.

Rosato R.R., Almenara J.A.,Yu C. et al.: Evidence of a functional role for $\mathrm{p} 21^{\mathrm{WAF} 1 / \mathrm{Cip} 1}$ downregulation in synergistic antileukemic interactions between the histone deacetylase inhibitor sodium butyrate and flavopiridol. Mol. Pharmacol. 65:571-581, 2004.
Suenaga M., Soda H., Oka M. et al.: Histone deacetylase inhibitors suppress telomerase reverse transcriptase mRNA expression in prostate cancer cells. Int. J. Cancer 97:621625, 2002.

Vrana J.A., Decker R.H., Johnson C.R. et al.: Induction of apoptosis in U 937 human leukemia cells by suberoylanilide hydroxamic acid (SAHA) proceeds through pathways that are regulated by $\mathrm{Bcl}-2 / \mathrm{Bcl}-\mathrm{XL}$, c-Jun, and p21CIP1, but independent of p53. Oncogene 18:7016-7025, 1999.

Warrell R.P., He L.Z., Richon V. et al.: Therapeutic targeting of transcription in acute promyelocytic leukemia by use of an inhibitor of histone deacetylase. J. Natl. Cancer Inst. 90:1621-1925, 1998.

Wu J.T., Archer S.Y., Hinnebusch B. et al.: Transient vs. prolonged histone hyperacetylation: effects on colon cancer cell growth, differentiation, and apoptosis. Am. J. Physiol. Gastrointest. Liver Physiol. 280:G482-G490, 2001.

Yoshida M., Kijima M., Akita M. et al.: Potent and specific inhibition of mammalian histone deacetylase both in vivo and in vitro by trichostatin A. J. Biol. Chem. 265:1717417179, 1990.

Yoshida M., Furumai R., Nishiyama M. et al..: Histone deacetylase as a new target for cancer chemotherapy. Cancer Chemother. Pharmacol. 48(Suppl.11):20-26, 2001. 